\title{
Decompressive craniectomy for traumatic brain injury: outcomes and their determinants
}

\author{
Jeewan Rankothkumbura ${ }^{1}$, Hemantha Gunathilaka ${ }^{1}$, Saman Wadanamby ${ }^{1}$
}

\begin{abstract}
Background Decompressive craniectomy (DC) is a management option to control raised intracranial pressure (ICP) in traumatic brain injury (TBI) with inconsistent evidence for its outcomes and their determinants.
\end{abstract}

Objectives The aim of this study was to assess the outcomes and determinants of outcomes of DC done in National Hospital of Sri Lanka (NHSL) at one year and three years of follow-up.

Materials and methods Patients who underwent DC for TBI within 6 months period from 01/02/2016 to 31/07/ 2016 at the Neurotrauma Centre, NHSL were included in the study. Data were retrieved from medical records. Outcomes were evaluated by interviewing patients/ relatives over the telephone using standard questionnaire for extended Glasgow Outcome scale (GOS-E).

Results Inclusion and exclusion criteria matched 118 patients were selected and $89(75.42 \%)$ contactable patients were included in the analysis. Majority (86.4\%) were males and median age was 45 years. There were 56 primary DCs and 33 secondary DCs. Favorable outcomes (GOS-E 5-8) were seen in $20.2 \%$ and in $24.7 \%$ at the end of one year and three years respectively. Younger age, good pupillary reaction and higher GCS on admission were associated with statistically significant favorable outcomes $(P<0.05)$. Pupillary symmetry, timing of DC (primary or secondary), time elapsed from time of injury to performing primary DC, type of DC, whether CT shows an isolated lesion or multiple lesions, submission to tracheostomy, having medical comorbidities and postoperative infections were not predictive of the outcome.

Conclusion Favorable functional outcomes following DC for TBI is limited to $20-25 \%$. Younger age, good pupillary reaction and higher GCS are predictors of favorable functional outcomes.

Ceylon Medical Journal 2021; 66: 32-37

DOI: http://doi.org/10.4038/cmj.v66i1.9353

${ }^{1}$ Neurotrauma Centre, National Hospital of Sri Lanka.

\section{Introduction}

Traumatic brain injury (TBI) remains as one of the commonest cause of death in trauma [1 - 3]. With the light of many research activities in parallel to advancements in technology, management of TBI has evolved a lot, over the past few decades. Strategies of minimizing secondary brain injury have contributed to the improvement of mortality following TBI. The best proof for this is the reduction of mortality following severe TBI over last three decades from $50 \%$ to $25 \%$ [4]. Nonetheless, management of severe TBI still remains an immense challenge in neurosurgical field. Apart from barbiturate coma, decompressive craniectomy (DC) remains as an ultimate option in management of intractable intracranial hypertension in TBI. However there is a significant discrepancy in available literature regarding outcomes and their determinants of this procedure and yet no study has been published on this topic in the local context. Therefore objective of this study was to assess outcomes and the determinants of outcomes of this highly demanding surgical procedure. The results of this study will be important in selective use of DC.

\section{Methods and materials}

All patients who underwent DC for TBI over a 6 months period from 01/02/2016 to 31/07/2016 at the Neurotrauma Centre, National Hospital of Sri Lanka (NHSL) were selected for the study. Data was retrieved from patients' medical records by three investigators. Data on demographic factors, cause of injury, pre-intubation Glasgow Coma Scale (GCS), pupillary symmetry and reaction at admission, comorbidities, computerized tomography (CT) findings; extradural haemorrhage (EDH), subdural haemorrhage (SDH), subarachnoid haemorrhage (SAH), cerebral contusion , type of DC (bifrontal DC, hemispheric craniectomy and posterior fossa DC), timing of DC (whether primary or secondary), time elapsed from time of injury to performing primary DC, duration of ICU/hospital stay, submission to tracheostomy, data on post-operative infections and other complications were collected.

Correspondence: JR, e-mail: <jeewan.med@gmail.com>. Received 01 November 2020 and revised version 28 February 2021 accepted 20 March 2021.

This is an open-access article distributed under the terms of the Creative Commons Attribution License, which permits unrestricted use, distribution, and reproduction in any medium, provided the original author and source are credited. 
Data were recorded in data collection forms consisted of check boxes to avoid observer bias. Patients with polytrauma and significant chest, abdomen or limb injuries were excluded to avoid confounding bias.

Outcomes were evaluated by interviewing patients/ relatives over the telephone. The patients/relatives were contacted through the contact numbers provided to hospital and the consent to recruit in the study was obtained after providing detailed information. This was done by a separate investigator who was blinded for the baseline data, using standard questionnaire for extended Glasgow Outcome scale (GOS-E) at one year and three year intervals, after excluding in-hospital mortality. We used GOS-E, which is the current gold standard to evaluate functional outcome. Studies have shown that structured interview format used to assign a GOS-E score provides more comprehensive assessment of disability [5]. Over the telephone structured interviews have been proven effective as a substitute for contact interviews by Pettigrew et al [6]. Therefore we utilized that method as the tool of outcome data collection. GOS-E 1 to 4 were categorized as unfavorable outcomes and 5 to 8 were categorized as favorable outcomes.

Data analysis was done using IBM SPSS statistics version 25. Chi square test, McNemar's test and t-test were used appropriately. Ethical clearance for the study was obtained from Ethical Review Committee, NHSL.

\section{Results}

A total of 124 DCs have been performed during the study period. Inclusion and exclusion criteria matched 118 patients were selected for the study. Out of this, 89 patients (75.42\%) who were contactable at the end of one year and three years were included in the analysis. There were no statistically significant differences on baseline characteristics such as age, sex, GCS at presentation, pupillary symmetry and reaction, comorbidities, CT findings and type and timing of DC between the dropout group and the group included in the analysis. Majority $(n=77,86.5 \%)$ were males and median age was 45 years (interquartile range 29.5 years to 60.5 years). Data on cause of injury, CT findings, timing and type DC and presence of medical comorbidities are depicted on Table 1.

DCs performed within 24 hours from the time of injury with evacuation of the mass lesion were defined as primary DCs and those performed after 24 hours from the time of injury typically to treat raised ICP following failed medical management were defined as secondary DCs.

Patients for DCs have been selected by the treating neurosurgeon, case by case based on clinical and radiological features. GCS $<13$ with mass lesion $>30 \mathrm{~mL}$ on CT (>15-20 mL in temporal fossa or posterior fossa) or asymmetrical and slow or nonreactive pupils, GCS $<8$ salvageable TBIs even without mass lesion on CT have been treated with primary DC.

Table 1. Characteristics of patients included in the study

\begin{tabular}{|c|c|}
\hline Characteristic & Number (\%) \\
\hline \multicolumn{2}{|l|}{ Cause of injury } \\
\hline Road Traffic Accidents & $67 \quad(75.3 \%)$ \\
\hline Falls & $13(14.6 \%)$ \\
\hline Assaults & $4(4.4 \%)$ \\
\hline Unknown mechanisms & $5(5.6 \%)$ \\
\hline \multicolumn{2}{|l|}{ CT findings } \\
\hline Isolated unilateral EDH/SDH & $10(11.2 \%)$ \\
\hline Bilateral or unilateral multiple intra-axial TBI & 79 (88.8\%) \\
\hline \multicolumn{2}{|l|}{ Timing of DC } \\
\hline Primary & $56 \quad(62.9 \%)$ \\
\hline Secondary & $33(37.1 \%)$ \\
\hline \multicolumn{2}{|l|}{ Type of DC } \\
\hline Bifrontal & 35 (39.3\%) \\
\hline Hemispheric craniectomy & $51(57.3 \%)$ \\
\hline Posterior fossa & $3(3.4 \%)$ \\
\hline \multicolumn{2}{|l|}{ Medical comorbidities } \\
\hline At least one comorbidity & $16(18 \%)$ \\
\hline No comorbidities & 73 (82\%) \\
\hline
\end{tabular}


Medical management strategies provided to reduce raised ICP were, elevation of head end to $30^{\circ}$, removal of cervical collar, sedation of the patient, provision of adequate analgesia, maintenance of optimal physiological parameters for TBI $\left(\mathrm{PaO}_{2}>100 \mathrm{mmHg}, \mathrm{PaCO}_{2} 37-40 \mathrm{mmHg}\right.$, $\mathrm{pH}$ 7.35-7.45, systolic blood pressure $>110 \mathrm{mmHg}$, normothermia and normoglycaemia) and maintenance of serum sodium level around $145 \mathrm{mmol} / \mathrm{L}$ with infusion of $3 \%$ saline $0.5-1 \mathrm{~mL} / \mathrm{kg} / \mathrm{h}$ over $6-8$ hours. Medical management was considered as failed if GCS drops by 2 or more points with CT evidence of cerebral herniation or ICP remains $>20 \mathrm{mmHg}$ for more than 15 minutes within an hour (in whom ICP was monitored). These patients have been treated with secondary DC. ICP has been monitored only in 16 patients in this group due to limited resources.

Median hospital stay of a patient at NHSL was 9 days (range 1-144 days) and median stay in ICU was 5 days (range 1 to 54 days). Thirty eight (42.7\%) patients died in the hospital and 39 patients (43.8\%) were transferred to local hospitals for continuing care while only 12 patients (13.5\%) were sent home directly. Thirty nine patients (43.8\%) suffered some sort of infection during hospital stay; majority $(n=23,25.8 \%)$ were respiratory tract infections while 5 (5.6\%) patients got central nervous system infections. In addition to post-operative infections, the following complications were observed; haemorrhage in 2 (2.25\%), seizures in 4 (4.5\%), subdural collections in 4 (4.45\%), cerebrospinal fluid leak in 2 (2.25\%) and obstructive hydrocephalus requiring ventriculoperitoneal shunt insertion in 4 (4.5\%). Re-operation or secondary surgical procedures were required in $14(15.73 \%)$ patients and tracheostomy was done in 52 (58.4\%) patients.

At the end of one year 25 (28\%) patients were alive and only one patient has died within next two years. Out of 25 survivors at one year, 21 (84\%) patients underwent cranioplasty (8 autologous and 13 titanium mesh). GOS-E values at the end of one year and three years were as depicted in Table 2.

Age less than 45 years and good pupillary reaction at the time of admission were associated with favorable outcomes. Female sex was associated with favorable outcomes only at the end of one year $(\mathrm{P}=0.047)$. Preintubation GCS has shown a significant association with outcomes only after three years $(\mathrm{P}=0.043)$. Pupillary symmetry, timing of DC, type of DC, submission to tracheostomy, CT findings, presence of comorbidities, post-operative infections did not show statistically significant association for outcomes (Table 3).

Mean time to start primary DC, from the time of injury was 7.5 hours. There was no significant association between time elapsed from the time of injury to performing $\mathrm{DC}$ and the outcome at one year or at three years $(\mathrm{P}=0.490$ and 0.4260 respectively).

In a subgroup analysis (after excluding dead, vegetative state and upper good recovery) to find out differences in GOS-E at end of one year and three year time, there was a statistically significant difference $(\mathrm{P}=0.05)$ indicating an improvement of patients' outcomes with time.

Table 2. GOS-E at end of one year and three years

\begin{tabular}{|c|c|c|c|c|c|}
\hline$G O S-E$ & & At one year & & At three years & \\
\hline & 1 - Dead & $64(71.9 \%)$ & & $65(73.0 \%)$ & \\
\hline & 2 - Vegetative state & 0 & & 0 & \\
\hline \multirow[t]{2}{*}{ Unfavorable outcome } & 3 - Lower severe disability & $3(3.4 \%)$ & $71(79.8 \%)$ & 0 & $67(75.3 \%)$ \\
\hline & 4 - Upper severe disability & $4(4.5 \%)$ & & $2(2.3 \%)$ & \\
\hline \multirow[t]{5}{*}{ Favorable outcome } & 5 - Lower moderate disability & $3(3.4 \%)$ & & $5(5.6 \%)$ & $22(24.7 \%)$ \\
\hline & 6 - Upper moderate disability & $3(3.4 \%)$ & $18(20.2 \%)$ & 0 & \\
\hline & 7 - Lower good recovery & $5(5.6 \%)$ & & $9(10.1 \%)$ & \\
\hline & 8 - Upper good recovery & $7(7.9 \%)$ & & $8(9.0 \%)$ & \\
\hline & & $89(100 \%)$ & & $89(100 \%)$ & \\
\hline
\end{tabular}


Table 3. Analysis of outcomes ( ${ }^{a}$ - 11 cases excluded due to incomplete data, b - only bifrontal and hemispheric DCs considered for analysis)

\begin{tabular}{|c|c|c|c|c|c|c|c|}
\hline \multirow[b]{2}{*}{ Factor/Independent variable } & & \multicolumn{3}{|c|}{ At one year } & \multicolumn{2}{|c|}{ At three years } & \multirow[b]{2}{*}{$\begin{array}{c}\text { Significance } \\
(P \text {-value })\end{array}$} \\
\hline & & $\begin{array}{l}\text { Unfavorable } \\
\text { outcomes }\end{array}$ & $\begin{array}{l}\text { Favorable } \\
\text { outcomes }\end{array}$ & $\begin{array}{l}\text { Significance } \\
\text { (P-value) }\end{array}$ & $\begin{array}{l}\text { Unfavorable } \\
\text { outcomes }\end{array}$ & $\begin{array}{c}\text { Favorable } \\
\text { outcomes }\end{array}$ & \\
\hline \multirow[t]{2}{*}{ Age } & $<45 y$ & 32 & 17 & $0.000 *$ & 32 & 17 & $0.016 *$ \\
\hline & $>45 y$ & 39 & 1 & & 35 & 5 & \\
\hline \multirow[t]{2}{*}{ Sex } & Male & 64 & 13 & $0.047^{*}$ & 60 & 17 & 0.143 \\
\hline & Female & 7 & 5 & & 7 & 5 & \\
\hline \multirow[t]{2}{*}{ Pupillary symmetry ${ }^{\mathrm{a}}$} & Symmetrical & 38 & 11 & 0.582 & 35 & 14 & 0.441 \\
\hline & Asymmetrical & 24 & 5 & & 23 & 6 & \\
\hline \multirow[t]{3}{*}{ Pupillary reaction ${ }^{\mathrm{a}}$} & Well reactive & 16 & 10 & $0.020^{*}$ & 13 & 13 & $0.002 *$ \\
\hline & Slow reactive & 19 & 3 & & 19 & 3 & \\
\hline & Non-reactive & 27 & 3 & & 26 & 4 & \\
\hline \multirow[t]{2}{*}{ Timing of DC } & Primary DC & 46 & 10 & 0.469 & 44 & 12 & 0.349 \\
\hline & Secondary DC & 25 & 8 & & 23 & 10 & \\
\hline \multirow[t]{2}{*}{ Type of $\mathrm{DC}^{\mathrm{b}}$} & Bifrontal & 26 & 9 & 0.366 & 25 & 10 & 0.599 \\
\hline & $\begin{array}{l}\text { Hemispheric } \\
\text { craniectomy }\end{array}$ & 42 & 9 & & 39 & 12 & \\
\hline \multirow[t]{2}{*}{ Tracheostomy } & Done & 45 & 7 & 0.060 & 41 & 11 & 0.355 \\
\hline & Not done & 26 & 11 & & 26 & 11 & \\
\hline \multirow[t]{2}{*}{ Severity of head injury - } & Severe & 35 & 5 & & 35 & 5 & \\
\hline & GCS 3-8 & & & & & & \\
\hline \multirow[t]{4}{*}{ GCS criteria } & Moderate & 17 & 6 & 0.260 & 14 & 9 & $0.043^{*}$ \\
\hline & GCS 9-12 & & & & & & \\
\hline & Mild & 19 & 7 & & 18 & 8 & \\
\hline & GCS 13-15 & & & & & & \\
\hline \multirow[t]{4}{*}{ CT findings } & Unilateral isolated & 7 & 3 & 0.414 & 6 & 4 & 0.234 \\
\hline & EDH/SDH & & & & & & \\
\hline & Bilateral/Multiple & 64 & 15 & & 61 & 18 & \\
\hline & TBI & & & & & & \\
\hline Presence of medical & None & 56 & 17 & 0.124 & 53 & 20 & 0.211 \\
\hline comorbidities & Present & 15 & 1 & & 14 & 2 & \\
\hline \multirow[t]{2}{*}{ Post-operative infections } & None & 46 & 14 & 0.294 & 45 & 15 & 0.930 \\
\hline & Any & 25 & 4 & & 22 & 7 & \\
\hline
\end{tabular}

\section{Discussion}

Many of the currently available studies have evaluated outcomes of DC at 3 or 6 months period. However, morality rate following DC is highly variable in literature (11\% to 59\%) [7]. In this study we have evaluated the outcome of DC at one year and 3 year intervals, providing insight into long term outcomes as well. In our series mortality remains at significantly higher rate which is $71.3 \%$ at one year and $74.64 \%$ at three years compared to other studies. However when results were separately analyzed according to GOS-E categorizes as unfavorable (GOS-E 1-4) and favorable (GOS-E 5-8), our results are relatively comparable with other studies including the landmark DECRA trial and RESCUEicp trial which report $70 \%$ unfavorable outcomes at 6 months [8-10]. Similarly 
after 49+/-25 months of mean follow up, U. Mier et al has also reported mortality of $57 \%$ and $75 \%$ unfavorable outcomes (Glasgow Outcome Scale 1-3) following DC as a long term follow up study [11].

In our study, apart from the two extremes of GOS-E spectrum (i.e. dead or vegetative and good recovery) those who were at moderate to severe disability have shown significant difference in their disability level at one year and three years of follow up, showing improvement of GOS-E over time $(\mathrm{P}=0.05)$. This indicates need of continuous follow up care for these patients.

Comparative to our study many studies report, younger age as a determinant of favorable outcomes than the older age, though the age cut off limits are heterogeneous among studies [12-14]. Similarly, good pupillary reaction is a significant predictor of favorable outcomes in our study as well in other published studies [10, 14, 15]. However pupillary symmetry has not shown any predictive value on the outcome. Although in our study female sex was associated with favorable outcomes at the end of one year, this finding was not supported in literature. This may be attributed to the small number of female participants in the study. Pre-intubation GCS has been reported as a significant predictor of the outcome in several studies $[12,14,15]$. A comparable finding was observed in our study although it is statistically significant only at the end of 3 years.

Al-Jishi et al has reported secondary DC has significantly favorable outcomes than primary DC, which is in contrary to our finding [7]. However it should be noted that the decision on secondary DC was sometimes made clinically rather than based on ICP measurements due to lack of ICP monitoring facility in our setup. Even so, the systematic review and meta-analysis by Fathima et al reports that there is no significant difference in early or late DC with regard to outcomes based on three studies [16].

Performing tracheostomy has not shown any association on outcome in our study. However Laghari et al has reported tracheostomy as a negative predictor of good functional outcome [13]. Saade et al has reported that having multiple lesions on CT results in poor outcomes compared to presence of a single lesion [10]. However our finding is that there is no association between presence of a single lesion vs multiple or bilateral lesions with the outcome. Some studies state midline shift as a poor predictor of functional outcome [10, 12, 14]. However according to Gouello et al, Marshal Score which includes midline shift as a determinant is not suitable as a predictive factor for outcome following TBI [15].

Our study has shown that presence of medical comorbidities or post-operative infections as a complication do not have any association with the outcome following DC. This is a new evidence with a great importance on clinical decision making.
Owing to retrospective nature of the sample collection, non-comparison of the DC group with a case matched non-DC group is a limitation of this study.

It should also be noted that even with extensive research including randomized trials such as DECRA and RESCUEicp, DC remains as a controversial management option and there are significant lapses in evidence on various aspects of DC and its outcome [17]. Therefore high quality studies with long term follow up periods are essential to fill these evidence gap.

In conclusion, this study supports the existing evidence; younger age, good pupillary reaction and higher GCS at presentation as predictive factors for favorable outcomes following DC for patients with TBI. This emphasize that the clinical decisions should be based on these evidence in order to yield favorable outcomes in this highly demanding surgical procedure, especially such as in our setup due to limited resources. Similarly DC should not be performed as the default option to manage raised ICP following TBI.

\section{Abbreviations}

DC: Decompressive craniectomy

ICP: Intracranial pressure

TBI: Traumatic Brain Injury

NHSL: National Hospital of Sri Lanka

GOS-E: Extended Glasgow Outcome Scale

GCS: Glasgow Coma Scale

CT: Computerized Tomography

EDH: Extradural haemorrhage

SDH: Subdural haemorrhage

SAH: Subarachnoid haemorrhage

\section{Declarations}

Authors' contributions: JR conceived the study and led the proposal, collection, entry and analysis of data and the study report. HG involved in data collection and data entry. SW guided the study conduct and edited the proposal and the study report.

Competing interests: The authors declare that they have no competing interests

Ethical approval: Ethical approval was obtained from the Ethics Review Committee of the NHSL (ETH/COM/2017/ 11).

Funding: None.

\section{Acknowledgements}

We thank Dr. T. H. T. Thoradeniya, Dr. P. C. Amadoruge and Dr. A. L. A. Shiyam for their contribution for data collection and Dr. G. J. Chandana for data analysis. 


\section{References}

1. Baker CC, Oppenheimer L, Stephens B, Lewis FR, Trunkey DD. Epidemiology of trauma deaths. Am J Surg. 1980; 140(1): 144-50.

2. Shackford SR, Mackersie RC, Holbrook TL, et al. A population-based analysis. Arch Surg. 1993; 128(5): 571-5.

3. Acosta JA, Yang JC, Winchell RJ, et al. Lethal injuries and time to death in a level I trauma center. J Am Coll Surg. 1998; 186(5): 528-33.

4. Brain Trauma Foundation; American Association of Neurological Surgeons; Congress of Neurological Surgeons. Guidelines for the management of severe traumatic brain injury. J Neurotrauma 2007; 24 Suppl 1: S1-106.

5. Pettigrew LEL, Wilson JTL, Teasdale GM. Assessing disability after head injury: improved use of the Glasgow Outcome Scale. J Neurosurg. 1998; 89(6): 939-43.

6. Pettigrew LE, Wilson JT, Teasdale GM. Reliability of ratings on the Glasgow Outcome Scales from in-person and telephone structured interviews. J Head Trauma Rehabil. 2003; 18(3): 252-8.

7. Al-Jishi A, Saluja RS, Al-Jehani H, Lamoureux J, Maleki M, Marcoux J. Primary or secondary decompressive craniectomy: different indication and outcome. Can J Neurol Sci. 2011; 38(4): 612-20.

8. Cooper DJ, Rosenfeld JV, Murray L, et al. Decompressive craniectomy in diffuse traumatic brain injury. $N$ Engl J Med. 2011; 364(16): 1493-502.

9. Hutchinson PJ, Kolias AG, Timofeev IS, et al. Trial of Decompressive Craniectomy for Traumatic Intracranial Hypertension. N Engl J Med. 2016; 375(12): 1119-30.

10. Saade N, Veiga JCE, Cannoni LF, et al. Evaluation of prognostic factors of decompressive craniectomy in the treatment of severe traumatic brain injury. Rev Colégio Bras Cir. 2014; 41(4): 256-62.

11. Meier U, Ahmadi S, Killeen T, Al-Zain FT, Lemcke J. Longterm outcomes following decompressive craniectomy for severe head injury. Acta Neurochir Suppl. 2008; 102: 29-31.

12. Kuo JR, Lo CJ, Lu CL, Chio CC, Wang CC, Lin KC. Prognostic Predictors of Outcome in an Operative Series in Traumatic Brain Injury Patients. J Formos Med Assoc. 2011; 110(4): 258-64.

13. Laghari AA, Bari ME, Waqas M, Ahmed SI, Nathani KR, Moazzam W. Outcome of Decompressive Craniectomy in Traumatic Closed Head Injury. Asian J Neurosurg. 2018; 13(4): 1053-6.

14. Sinha S, Raheja A, Garg M, et al. Decompressive craniectomy in traumatic brain injury: A single-center, multivariate analysis of 1,236 patients at a tertiary care hospital in India. Neurol India 2015; 63(2): 175.

15. Gouello G, Hamel O, Asehnoune K, Bord E, Robert R, Buffenoir K. Study of the long-term results of decompressive craniectomy after severe traumatic brain injury based on a series of 60 consecutive cases. Scientific World Journal 2014; 2014: 207585.

16. Fatima N, Rumaihi GA, Shuaib A, Saqqur M. The role of decompressive craniectomy in traumatic brain injury: A systematic review and meta-analysis. Asian J Neurosurg 2019; 14(2): 371.

17. Hawryluk GWJ, Rubiano AM, Totten AM, et al. Guidelines for the Management of Severe Traumatic Brain Injury: 2020 Update of the Decompressive Craniectomy Recommendations. Neurosurgery 2020; 87(3): 427-34. 